\title{
Understanding the Disease Course and Therapeutic Benefit of Tafamidis Across Real-World Studies of Hereditary Transthyretin Amyloidosis with Polyneuropathy: A Proof of Concept for Integrative Data Analytic Approaches
}

\author{
Daniel Serrano $\cdot$ Christopher B. Atzinger · Marc F. Botteman
}

Received: February 21, 2018 / Published online: April 2, 2018

(C) The Author(s) 2018

\begin{abstract}
Introduction: Hereditary transthyretin (TTR) amyloidosis with polyneuropathy (hATTR-PN) is a rare, autosomal dominant amyloidosis characterized primarily by progressive ascending sensorimotor neuropathy often associated with autonomic involvement. hATTR-PN is caused by a mutation in the TTR gene leading to protein misfolding and amyloid accumulation in peripheral nerves and vital organs. The latest global prevalence estimates point to 10,000 cases worldwide, with an upper end of about 40,000. Tafamidis has been approved in over 40 countries for delaying neurologic disease progression in early-stage hATTR-PN. Multiple observational studies have examined clinical outcomes in hATTR-PN patients treated with tafamidis in the routine clinical setting. Integrative data analysis (IDA) is a technique for optimally constructing synthetic treatment and control cohorts from multiple independent studies, which allows meta-analysis of patient-level data. Herein, we provide a proof of concept for the application of IDA to real-world and natural history hATTR-PN
\end{abstract}

Enhanced content To view enhanced content for this article go to https://doi.org/10.6084/m9.figshare.59997 56.

D. Serrano · C. B. Atzinger · M. F. Botteman ( $ه)$ Pharmerit International, 4350 East-West Highway, Suite 1110, Bethesda, MD 20814, USA e-mail: mbotteman@pharmerit.com data. IDA permits increased understanding of outcomes in tafamidis-treated and untreated persons with hATTR-PN by optimally pooling all available information.

Methods: Summary statistics corresponding to the Neuropathy Impairment Score-Lower Limb (NIS-LL) from five published studies were pooled, converted to change from baseline means and variances, and analyzed using IDA. IDA-based synthetic cohorts were generated by averaging across studies stratified on treatment versus control cohort. Trends in change from baseline in each study and the corresponding synthetic cohorts were plotted. Patient-level data were simulated from the synthetic cohort trends in a Monte Carlo simulation to highlight the ability to contrast synthetic cohort trends using the mixed model for repeated measures (MMRM).

Results: The average sample size among the five studies was 71 (37-128) patients. The average NIS-LL trends indicated that tafamidistreated patients experienced slower progression in neuropathy compared to untreated patients. Synthetic cohort trends reflected the trends observed in the contributing studies, while simultaneously shrinking the width of corresponding confidence bands. Monte Carlo simulation results demonstrated precise recovery of the synthetic cohort and time-dependent simulated NIS-LL means by the MMRM.

Discussion: This proof of concept demonstrates the utility of IDA-based synthetic cohorts for 
increased precision in characterizing and testing hypotheses about treatment outcomes and prognosis in hATTR-PN.

Funding: Pfizer.

Plain Language Summary: Plain language summary available for this article.

\section{PLAIN LANGUAGE SUMMARY}

Hereditary transthyretin (TTR) amyloidosis with polyneuropathy (hATTR-PN) is a rare inherited disease. It is caused by genetic mutations that change the structure of TTR proteins, causing an abnormal buildup of amyloid protein deposits (amyloidosis) in your body's nerves and organs. This damages your nerves and organs and causes weakness, numbness, and pain. The drug tafamidis stabilizes TTR proteins and slows the disease's progression. Tafamidis has been approved in over 40 countries based on clinical trial results. However, researchers and doctors are still studying how it works for patients in the real world. Over the past few years, researchers have published multiple real-world studies about tafamidis, but it is difficult to get a full, uniform picture of how well tafamidis works, because the studies are too different. hATTR-PN is a rare disease, so the number of patients per study is small; small sample sizes can make it more difficult to tell true effects from statistical noise, whereas large samples can be more precise. Integrative data analysis (IDA) is a technique that allows researchers to combine the results of multiple studies into a large pool of data and analyze the larger data set (or "synthetic cohort") instead. This makes optimal use of each study's information and creates a fuller picture of the treatment's real-world results. This manuscript is a "proof of concept" to demonstrate how the IDA statistical method can be used to build synthetic cohorts from realworld hATTR-PN data, improving our understanding of hATTR-PN patients' outcomes.

Keywords: Integrated data analysis; Hereditary transthyretin amyloidosis; Meta-analysis; Tafamidis

\section{INTRODUCTION}

Hereditary transthyretin (TTR) amyloidosis with polyneuropathy (hATTR-PN) is a rare, autosomal dominant, systemic amyloidosis that is characterized primarily by progressive ascending sensorimotor neuropathy, with or without autonomic involvement, although mixed phenotypes are common $[1,2]$. The central estimate of global hATTR-PN prevalence is approximately 10,000 persons, but it may be as high as 40,000 [3]. hATTR-PN is traditionally categorized as either Val30Met [4] or non-Val30Met. The former is the most common variant globally [5]. These mutations make TTR tetramers prone to dissociating into monomers that undergo misfolding due to their physical structure; the misfolded proteins aggregate into insoluble amyloid fibrils that are deposited on peripheral nerves and in vital organs, leading to the symptoms of hATTR-PN. If untreated, the average survival is $10-15$ years after symptom onset [5-8].

Tafamidis is a selective TTR stabilizer that holds TTR tetramers together to prevent formation of misfolded TTR, and is approved in over 40 countries to delay neurologic disease progression in early-stage hATTR-PN [9]. The tafamidis clinical development program demonstrated the drug's long-term safety and effectiveness in delaying hATTR-PN disease progression for up to 5.5 years [10-15], with comparable outcomes observed in Val30Met and non-Val30Met patients compared to placebo [16].

Disease progression in hATTR-PN is typically measured according to standardized staging criteria that reflect the severity of systematic neurological involvement. One of the most frequently used staging systems is the polyneuropathy disability (PND) score [17], which ranges from stage 0 (no impairment) to stage IV (confined to a wheelchair or bedridden).

Since the initial approval of tafamidis in 2011 by the European Medicines Agency [18], various observational open-label studies have assessed its effectiveness among samples composed predominantly of stage I patients in the 
routine clinical (i.e., "real world") setting [10, 19-21]. Several key characteristics were consistent across these studies, including the assessments used to measure neuropathy progression and the duration of assessment intervals. However, the mutant variant distributions, ages of onset, and timing of treatment initiation relative to disease stage differed among the studies. This inter-study heterogeneity-in addition to small sample sizes, different analytical approaches, and variable follow-up times-has made it difficult to interpret the uniformity of the effect of tafamidis on hATTRPN progression. Key unresolved questions include whether progression and treatment response differ between mutation type, age of onset, and/or disease staging schemes.

We present herein a proof-of-concept study and applied example of a statistical method that can be used to pool real-world and randomized trial tafamidis study data. Methodological details are provided in an overview, and an applied example is described. The example constructs synthetic cohorts from summary statistics reported in the literature, and then contrasts the generated synthetic cohorts in a mixed model for repeated measures (MMRM) to characterize therapeutic benefit.

\section{METHODS}

Integrative data analysis (IDA) [22] is a statistical pooling method to combine studies and then construct synthetic treatment and control cohorts. Aggregation of heterogeneous studies into synthetic cohorts can be thought of as a meta-analytic technique for raw data. Optimal weighting and scaling techniques are used to produce a synthetic cohort from each individual study that up-weights each study's unique and usable information while simultaneously down-weighting its idiosyncratic noise. Available optimal pooling techniques range from the use of fixed and random study effects to inverse probability weighting (IPW) through propensity methods. This produces a synthetic cohort that is maximally representative of each study's useful information. These techniques can be used to aggregate data from treatment studies and control/natural history studies to create synthetic treatment and control arms. These synthetic treatment and control cohorts can then be contrasted to determine the time-dependent value of therapeutic intervention. These synthetic cohorts yield greater precision by increasing sample size while shrinking error variance.

Four extant studies were selected for analysis in addition to the tafamidis registration study, because each was among the largest recent studies and contributed comprehensive examinations of the relationship between disease progression and tafamidis treatment in a manner commensurate with the approach taken in the registration pivotal study [23]. Note that only three of the four were completely independent samples, and that the Coelho et al. [10] cohort considered here was the tafamidis crossover extension of the placebo arm in the registration trial. Table 1 provides a summary of these studies.

The five included studies were used to characterize study-specific trends in average change from baseline in Neuropathy Impairment ScoreLower Limb (NIS-LL) scores. In addition, the study-specific trends were averaged within treatment arms to construct synthetic cohorts for treatment and controls (i.e., natural history cohorts). Averages were used to construct the synthetic cohort trends because more sophisticated pooling procedures are not available with summary data. The averaging procedure was used to serve as a proof of concept for a forthcoming work in which patient-level data from some of the studies described herein (as well as others) will be pooled to create synthetic cohorts for direct analysis of treatment versus control/natural history cohorts.

Summary statistics reported in each of the included studies were used to obtain or construct study-stratified change from baseline means and corresponding confidence limits. In some studies-notably the 2012 registration study by Coelho et al. [23]—change from baseline means and 95\% confidence limits were not tabulated but rather were presented in figures only. In such cases, tracing software was used to recover as precisely as possible the numerical values presented in the figure. For the 
Table 1 Summary of trial-based and real-world prospective studies in patients with hATTR-PN treated with tafamidis

\begin{tabular}{|c|c|c|c|c|c|}
\hline & Cortese et al. [19] & Coelho et al. [23] & $\begin{array}{l}\text { Planté- } \\
\text { Bordeneuve } \\
\text { et al. [21] }\end{array}$ & Lozeron et al. [20] & Coelho et al. $[10]^{\mathrm{a}}$ \\
\hline $\begin{array}{l}\text { Study } \\
\text { design }\end{array}$ & $\begin{array}{l}\text { Multicenter, } \\
\text { observational }\end{array}$ & $\begin{array}{l}\text { Multicenter, } \\
\text { interventional }\end{array}$ & $\begin{array}{l}\text { Single center, } \\
\text { observational }\end{array}$ & $\begin{array}{l}\text { Single center, } \\
\text { observational }\end{array}$ & $\begin{array}{l}\text { Multicenter, } \\
\text { interventional }\end{array}$ \\
\hline Country & Italy & Global & France & France & Global \\
\hline $\begin{array}{l}\text { No. of } \\
\text { patients }\end{array}$ & 61 & 125 & 43 & 37 & 33 \\
\hline Male & $69 \%$ & $\begin{array}{l}50 \% \text { tafamidis, } 43 \% \\
\text { placebo }\end{array}$ & $56 \%$ & $67 \%$ & $45 \%$ \\
\hline $\begin{array}{l}\text { Mean age } \\
\text { at onset, } \\
y\end{array}$ & 59 & $36^{\mathrm{b}}$ & 59 & $58^{\mathrm{b}}$ & $36^{\mathrm{c}}$ \\
\hline $\begin{array}{l}\text { Duration } \\
\text { of } \\
\text { disease, y }\end{array}$ & 3.4 & 3.4 & 3.3 & 4.0 & $3.1^{\mathrm{d}}$ \\
\hline Val30Met & $28 \%$ & $100 \%$ & $47 \%$ & $100 \%$ & $100 \%$ \\
\hline Stage I & $72 \%$ & NR & NR & $67 \%$ & NR \\
\hline $\begin{array}{l}\text { Follow-up, } \\
\text { mo }\end{array}$ & 36 & 18 & 36 & 12 & 12 \\
\hline $\begin{array}{l}\text { Key } \\
\text { outcomes }\end{array}$ & $\begin{array}{l}\text { 33\% of pts remained } \\
\text { stable and did not } \\
\text { show significant } \\
\text { progression, } \\
\text { regardless of } \\
\text { mutation type and } \\
\text { baseline disease } \\
\text { stage } \\
\text { Neuropathy and } \\
\text { cardiomyopathy } \\
\text { progressed in a } \\
\text { proportion of pts } \\
\text { despite treatment }\end{array}$ & $\begin{array}{l}\text { In the efficacy } \\
\text { evaluable population } \\
(n=87), \\
\text { significantly more } \\
\text { tafamidis pts than } \\
\text { placebo pts had }<2 \\
\text { point NIS-LL } \\
\text { worsening from } \\
\text { baseline (60\% vs. } \\
38 \% ; p=0.041) \\
\text { Tafamidis pts had } \\
\text { better-preserved } \\
\text { TQOL }\end{array}$ & $\begin{array}{l}\text { At 6-12 mo, } \\
58 \%(25 / 43) \\
\text { of pts } \\
\text { showed a } \\
\text { response to } \\
\text { tafamidis } \\
\text { At } 30-36 \text { mo, } \\
9 \%(2 / 22) \\
\text { were still } \\
\text { stable }\end{array}$ & $\begin{array}{l}\text { At } 6 \text { mo, } 38 \% \text { of } 29 \\
\text { evaluable pts showed } \\
\text { no meaningful } \\
\text { progression in NIS- } \\
\text { LL (progression } \\
\text { defined as change } \\
\text { from baseline } \geq 2 \\
\text { points) } \\
\text { Of } 13 \text { pts evaluated at } \\
12 \text { mo, } 69 \% \text { had no } \\
\text { meaningful } \\
\text { progression in NIS- } \\
\text { LL }\end{array}$ & $\begin{array}{l}\text { In pts switched from } \\
\text { placebo, the } \\
\text { monthly rate of } \\
\text { change in NIS-LL } \\
\text { declined } \\
\text { Pts treated with } \\
\text { tafamidis for } 30 \text { mo } \\
\text { had } 55.9 \% \text { greater } \\
\text { preservation of } \\
\text { neurologic function } \\
\text { than pts in whom } \\
\text { tafamidis was } \\
\text { initiated later }\end{array}$ \\
\hline
\end{tabular}

hATTR-PN hereditary transthyretin amyloidosis with polyneuropathy, mo months, NIS-LL Neuropathy Impairment Score-Lower Limb, $N R$ not reported, pts patients, $T Q O L$ total quality of life, Val3OMet methionine replacement of valine at position 30 in the TTR gene, $y$ years

Notes

${ }^{a}$ Only the placebo-tafamidis arm was used from this study

b Age of onset computed from reported age minus disease duration

${ }^{c}$ Computed from reported median age minus median symptom duration

${ }^{\mathrm{d}}$ Median symptom duration 
control group, the only published data were from the placebo arm of the 18-month tafamidis registration study [23]. A separate cohort of controls was simulated that behaved in a manner consistent with our expectation of natural history disease progression in neuropathy. Specifically, this simulated cohort bore the characteristics of the 2012 registration study's placebo arm, except that it had worse progression (to reflect an assumed attenuation of any placebo effect) and it included a projection up to 30 months.

The outcome measure for this exercise was the average change from baseline in NIS-LL scores and corresponding 95\% confidence limits. However, not all studies reported NIS-LL scores in the change from baseline metric, nor did they necessarily report 95\% confidence limits when change from baseline means were available. Where average change from baseline and corresponding 95\% confidence limits were reported (i.e., Coelho et al. $[10,23]$ and Cortese et al. [19]), the statistics were used directly. Where time-specific NIS-LL means and standard deviations were reported (i.e., Lozeron et al. [20] and Planté-Bordeneuve et al. [21]), change from baseline means and standard deviations were computed using properties of the distribution for the difference in correlated Gaussian variables. Specifically, if Y1 and Y2 are correlated Gaussian vectors, with distribution $Y_{1} \sim N\left(\mu_{1}, \sigma_{1}^{2}\right)$ and $Y_{2} \sim N\left(\mu_{2}, \sigma_{2}^{2}\right)$, then $\left(Y_{2}-Y_{1}\right) \sim N\left(\mu_{2}-\mu_{1}, \sigma_{1}^{2}+\sigma_{2}^{2}+2 \rho \sigma_{1} \sigma_{2}\right)$, with corresponding standard deviation of $\mathrm{SD}_{\Delta}=\sqrt{\sigma_{1}^{2}+\sigma_{2}^{2}+2 \rho \sigma_{1} \sigma_{2}}$. In every case where change from baseline had to be constructed from time-dependent means and standard deviations, the correlation coefficient, $\rho$, was not reported. In these cases, $\rho$ was conservatively estimated as 0.4 for the purpose of approximating the difference standard deviation.

In the case of the Planté-Bordeneuve et al. [21] data, time-dependent means and standard deviations were reported for the NIS but not the NIS-LL. As the NIS-LL is a subset of the NIS, NIS-LL estimates were approximated from these summary statistics by scaling them to a range consistent with that observed for the other NIS-LL data. Specifically, the means were divided by 5.4 and the variances divided by 2.0.
For these data, the change from baseline statistics were computed as a function of the distribution for the difference in Gaussian variables scaled by a constant. Once the $\mathrm{SD}_{\Delta}$ was computed, 95\% Wald confidence limits were computed for the corresponding standard error. This procedure yielded change from baseline means $\left(\mu_{\Delta}\right)$ and corresponding $95 \%$ confidence limits. These estimates were averaged to construct the tabulated and plotted synthetic cohort average trend and corresponding 95\% Wald confidence limits, stratified by treatment arm.

Multivariate normal data were simulated from the synthetic cohort time-dependent treatment-arm-stratified means and variances using the "mvrnorm" function in $\mathrm{R}$ version 3.4.3 [24]. The simulated data were constructed under a balanced design with $n=100$ patients per cohort and complete data in repeated measures from month 6 through month 30 at 6 -month intervals. The variances were used to construct treatment-arm-stratified unstructured covariance matrices. In addition, correlated baseline covariates were simulated for both treatment arms generated to have a mean of 5 and a standard deviation of 2 . Simulated change from baseline data was modeled via the MMRM. This model was estimated using the MIXED procedure in SAS 9.4 software [25]. The model was parameterized using reference cell coding, treating the synthetic placebo as reference for the treatment effect and month 6 assessment as reference for time effect with continuous baseline covariate. Least squares means (LSMs) were estimated for each treatment by assessment level. The estimated LSMs were then plotted over the observed estimated synthetic cohort means to assess the model's ability to recover the observed assessment- and treatment-dependent means. This last component was conducted as part of the proof of concept to demonstrate that the model proposed for analysis of the final synthetic cohort data would successfully recover the functional form and observed means with acceptable precision. Fixed-effect point and interval estimates and variance components are not reported.

Tables were generated using the REPORT procedure in SAS 9.4 software, while figures were generated using the "ggplot2" package 
in $\mathrm{R}$ version 3.4.3. This article is based on previously conducted studies and does not contain any new data collected from human participants or animals.

This research is based on previously conducted studies and does not contain any studies with human participants or animals performed by any of the authors.

\section{RESULTS}

The reported or, in some cases, computed (e.g., the data computed for Planté-Bordeneuve [21]) values are tabulated for review in Table 2 (treated cohorts) and Table 3 (untreated or placebo cohorts). In addition, the estimates are plotted in several figures. Figure 1 presents the NIS-LL change from baseline trends stratified by study. Two clusters of trends are observed: the Cortese et al. and Lozeron et al. trends were comparable, and the Coelho et al. [23] and Planté-Bordeneuve et al. [21] trends were comparable. In all cases, the confidence bands were wide, reflecting in part the studies' small sample sizes, with the exception of Coelho et al. [23], which had a notably larger sample $(n=125)$ than the other studies (mean $n=57$ ). The only slightly outlying trend was associated with Coelho et al. [10]. However, the Coelho et al. [10] trend is distinct, since the original placebo cohort from the registration trial was switched to tafamidis treatment for the open-label continuation

Table 2 Study-stratified NIS-LL change from baseline means for tafamidis treatment cohorts

\begin{tabular}{lllllll}
\hline $\begin{array}{l}\text { Assessment } \\
\text { period }\end{array}$ & \multicolumn{6}{l}{ Mean (95\% confidence limits) NIS-LL change from baseline } \\
\cline { 2 - 7 } & $\begin{array}{l}\text { Coelho } \\
\text { et al. [23] }\end{array}$ & $\begin{array}{l}\text { Coelho } \\
\text { et al. [10] }\end{array}$ & $\begin{array}{l}\text { Lozeron } \\
\text { et al. [20] }\end{array}$ & $\begin{array}{l}\text { Cortese } \\
\text { et al. [19] }\end{array}$ & $\begin{array}{l}\text { Planté-Bordeneuve } \\
\text { et al. [21] }\end{array}$ & $\begin{array}{l}\text { Synthetic } \\
\text { treatment cohort }\end{array}$ \\
\hline Baseline & $0(0,0)$ & $0(0,0)$ & $0(0,0)$ & $0(0,0)$ & $0(0,0)$ & $0(0,0)$ \\
Month 6 & $1.3(0.7,1.8)$ & NA & $4.8(3.7,5.9)$ & $4.5(2.9,6.1)$ & $1.2(0.5,1.9)$ & $2.9(2.6,3.3)$ \\
Month 12 & $1.4(0.5,2.1)$ & NA & $6.6(3.8,9.4)$ & $5.9(3.6,8.2)$ & NA & $4.6(4.1,5.2)$ \\
Month 18 & $2.8(1.9,3.8)$ & NA & NA & $8.0(4.6,11.4)$ & $2.3(0.9,3.7)$ & $4.4(3.7,5.0)$ \\
Month 24 & $2.5(1.6,3.4)$ & $7.8(5.7,9.7)$ & NA & NA & NA & $5.1(4.4,5.9)$ \\
Month 30 & $3.0(1.5,4.4)$ & $6.8(5.1,8.5)$ & NA & NA & $4.3(2.4,6.2)$ & $4.7(4.3,5.1)$ \\
\hline
\end{tabular}

$N A$ not assessed (per study design), NIS-LL Neuropathy Impairment Score-Lower Limb

Table 3 Study-stratified NIS-LL change from baseline means for cohorts not receiving tafamidis

\begin{tabular}{llcc}
\hline Assessment period & \multicolumn{3}{l}{ Mean $(\mathbf{9 5 \%}$ confidence limits) change from baseline } \\
\cline { 2 - 4 } & Coelho et al. [23] & Natural history simulation & Synthetic control cohort \\
\hline Baseline & $0(0,0)$ & $0(0,0)$ & $0(0,0)$ \\
Month 6 & $2.0(1.5,2.7)$ & $3.2(2.4,4.0)$ & $2.6(2.4,2.8)$ \\
Month 12 & $4.7(3.9,5.6)$ & $6.2(5.9,6.6)$ & $5.5(5.2,5.7)$ \\
Month 18 & $5.8(4.9,6.8)$ & $7.9(7.4,8.5)$ & $6.9(6.6,7.1)$ \\
Month 24 & NA & $10.5(9.2,11.7)$ & $10.5(10.1,10.9)$ \\
Month 30 & NA & $12.8(11.4,14.1)$ & $12.8(12.3,13.3)$ \\
\hline
\end{tabular}

NA not assessed (per study design), NIS-LL Neuropathy Impairment Score-Lower Limb 


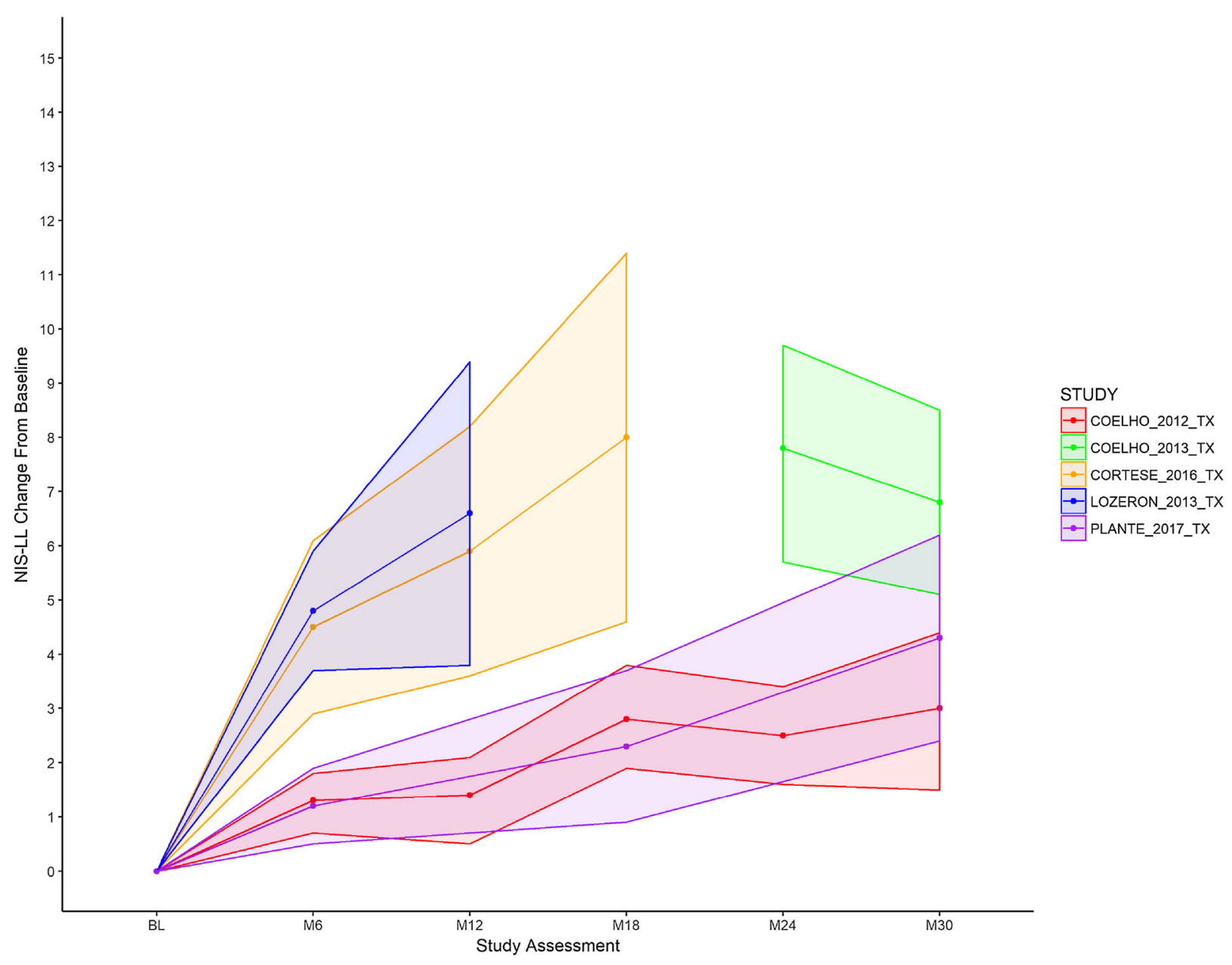

Fig. 1 Study-stratified mean (95\% confidence limits) NIS-LL change from baseline trend for tafamidis treatment cohorts. $B L$ baseline, $M$ month, NIS-LL Neuropathy Impairment Score-Lower Limb, TX treatment

study; the plotted trend is the change from baseline in NIS-LL scores post-crossover.

Broadly, the trends demonstrate a slowing of disease progression in NIS-LL associated with tafamidis. The average of these study-specific trends is presented in Fig. 2. The average trend, plotted in black, fits through the center of all study-specific trends, with a shape consistent with the Gompertz function suggested as appropriate for the NIS-LL data in hATTR-PN [26]. The same process was used to generate Figs. 3 and 4 for the Coelho et al. placebo arm [23] and the simulated natural history data. The treatment and placebo synthetic cohort trends were plotted together in Fig. 5. The trends overlap early, but as expected, diverge around month 12, as disease progression is uncontrolled in the untreated synthetic cohort and progression slows within the tafamidistreated synthetic cohort.

Within Fig. 6, the observed values and corresponding colors reported in Fig. 5 are retained (control = gray; tafamidis = black). These trends are overlaid with the model-estimated trends, which are also color-coded (control = orange; tafamidis = blue). As seen in Fig. 6, the observed synthetic cohort means (OBS) were precisely recovered by the MMRM-based values (LSMs). Notably, the discrepancy in estimates was zero between baseline and month 6 in both treatment and placebo synthetic cohorts, and zero between month 18 and month 24 for the placebo synthetic cohort. All other discrepancies were minor, and none evinced a departure from 


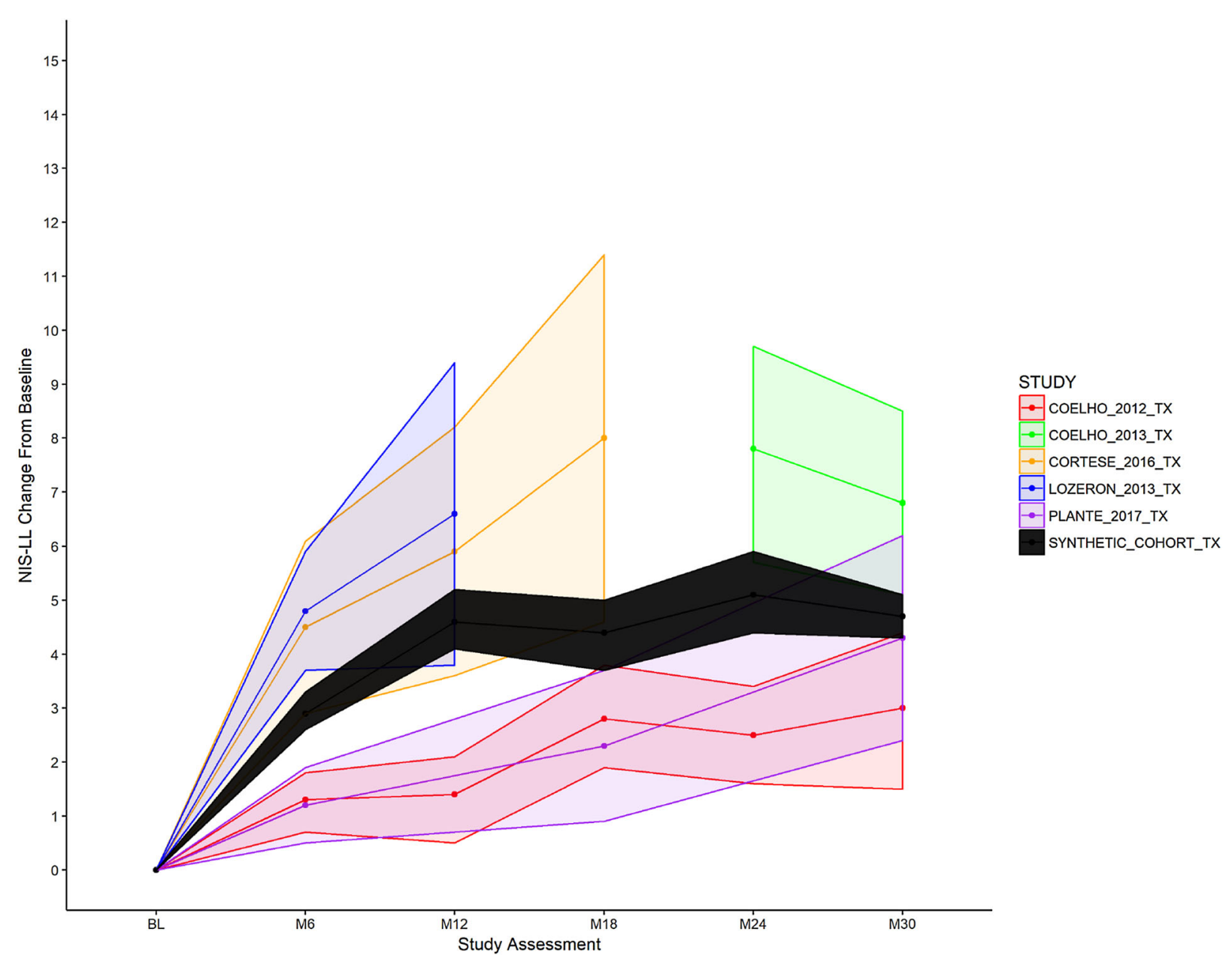

Fig. 2 Study-stratified mean (95\% confidence limits) NISLL change from baseline trend for tafamidis treatment cohorts, overlaying synthetic treatment cohort trend. $B L$

the observed functional form. Thus, the discrete-time MMRM is expected to precisely recover the observed means in the forthcoming analyses.

\section{DISCUSSION}

In this work, a synthetic cohort approach was applied to the analysis of real-world outcomes for tafamidis for the treatment of hATTR-PN, including comparison to natural history data. Our findings demonstrate the merits of employing synthetic cohorts. The average trend lines for the synthetic cohorts did not distort any of the study-specific trends. The error baseline, $M$ month, NIS-LL Neuropathy Impairment Score-Lower Limb, $T X$ treatment

variance, as measured by the width of the 95\% confidence bands, shrank relative to any individual study, but not excessively so. In addition, within the treatment synthetic cohort, the average trend and confidence bands mimicked a Gompertz function, which is a well-known function for modeling decelerating exponential effects that asymptote asymmetrically. This is of interest as the Gompertz function has been proposed elsewhere for the analysis of neurodegenerative outcome measures within hATTR-PN [26].

While the Gompertz function may be a good approximation to the average trend, one might encounter difficulty in properly specifying the model in the context of repeated measures and 


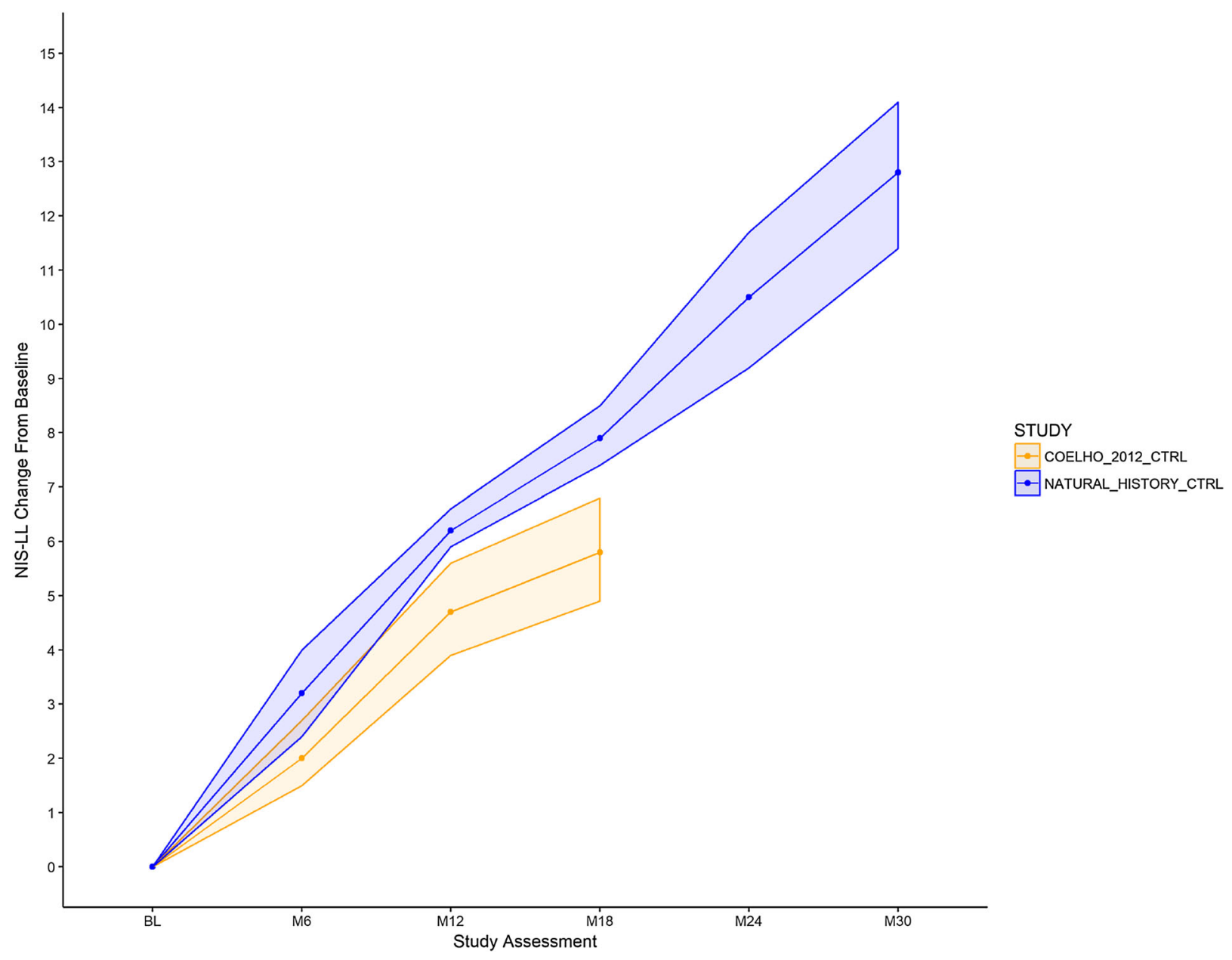

Fig. 3 Study-stratified mean (95\% confidence limits) NIS-LL change from baseline trend for natural history cohorts. $B L$ baseline, CTRL control, $M$ month, NIS-LL Neuropathy Impairment Score-Lower Limb

random effects. In contrast, a discrete-time MMRM is easily parameterized and can flexibly accommodate non-linear trends. Therefore, a second part of this proof of concept was to demonstrate that if individual-level data were simulated from synthetic cohort means and variances, a discrete-time MMRM could precisely recover the synthetic cohort-stratified mean trends. In fact, the discrete-time MMRM did succeed in recovering the observed means, pointing to the ability of this model to detect and accurately reflect synthetic cohort treatment arm differences in NIS-LL disease progression.

This proof-of-concept report has some limitations. The included studies may have had some overlap in the patient samples, which may have artificially limited the variance and caused the confidence limits to be underestimated. Summary statistics available in the literature were used, limiting the methods available for optimally weighting the pooling procedure averaging across studies.

In addition, three of the five studies considered were composed of samples that were 100\% Val30Met. The remaining two studies comprised mixed samples ( $<50 \%$ Val30Met). Published evidence has suggested that progression and treatment response outcomes differ substantially between Val30Met and non-Val30Met populations [27-29]. However, the recent analysis by Gundapaneni et al. [16] suggested that progression and treatment responses were no different between Val30Met and non-Val30Met 

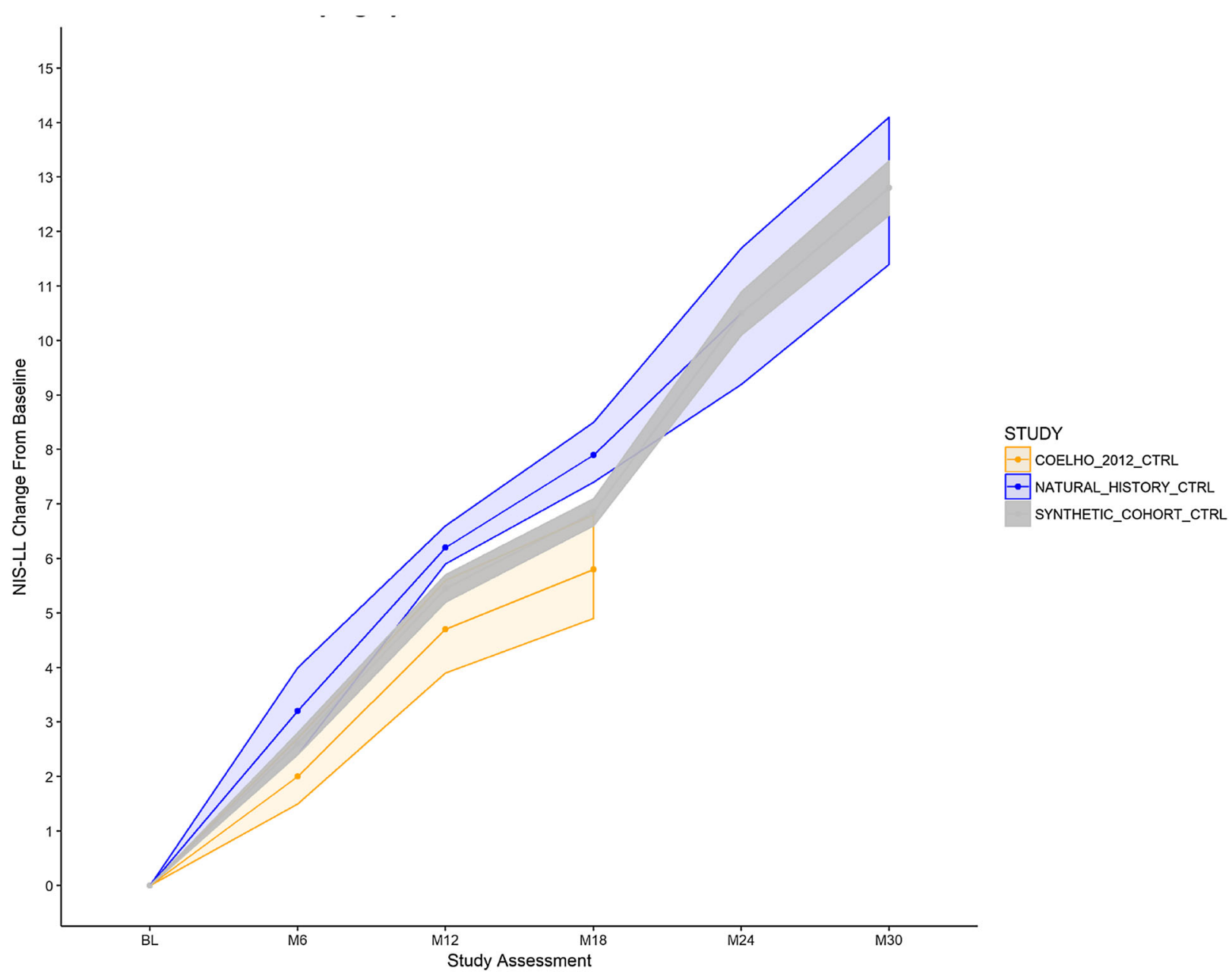

Fig. 4 Study-stratified mean (95\% confidence limits) NISLL change from baseline trend for non-tafamidis cohorts, overlaying synthetic control cohort trend. $B L$ baseline,

populations treated with tafamidis, after adjusting for baseline neuropathy status. A limitation of this proof-of-concept study is its inability to address the difference in progression and treatment response between these important sub-populations. However, given the modest sample sizes in the real-world data available to date, no single study has been able to do this either. As a consequence, given the findings of Gundapaneni et al., a new question that IDA may be uniquely positioned to answer is whether mutation-dependent progression and treatment differences are important, or whether they are artifacts arising from modest sample sizes and potentially insensitive analysis
CTRL control, $M$ month, NIS-LL Neuropathy Impairment Score-Lower Limb

techniques (i.e., responder analyses). It is our contention that a precise answer is likely achievable only by optimally pooling available data via IDA, and this issue speaks to the need to conduct this pooling research.

The next step in this line of research is to apply similar methods to the raw data corresponding to a larger set of real-world data studies. Doing so will allow for an IDA approach $[22,30]$ in which the patient-level data from a group of independent studies is pooled, rather than the aggregate. With patient-level data, more sophisticated and sensitive methods of pooling studies under optimal weighting paradigms can be employed. These include, but are 


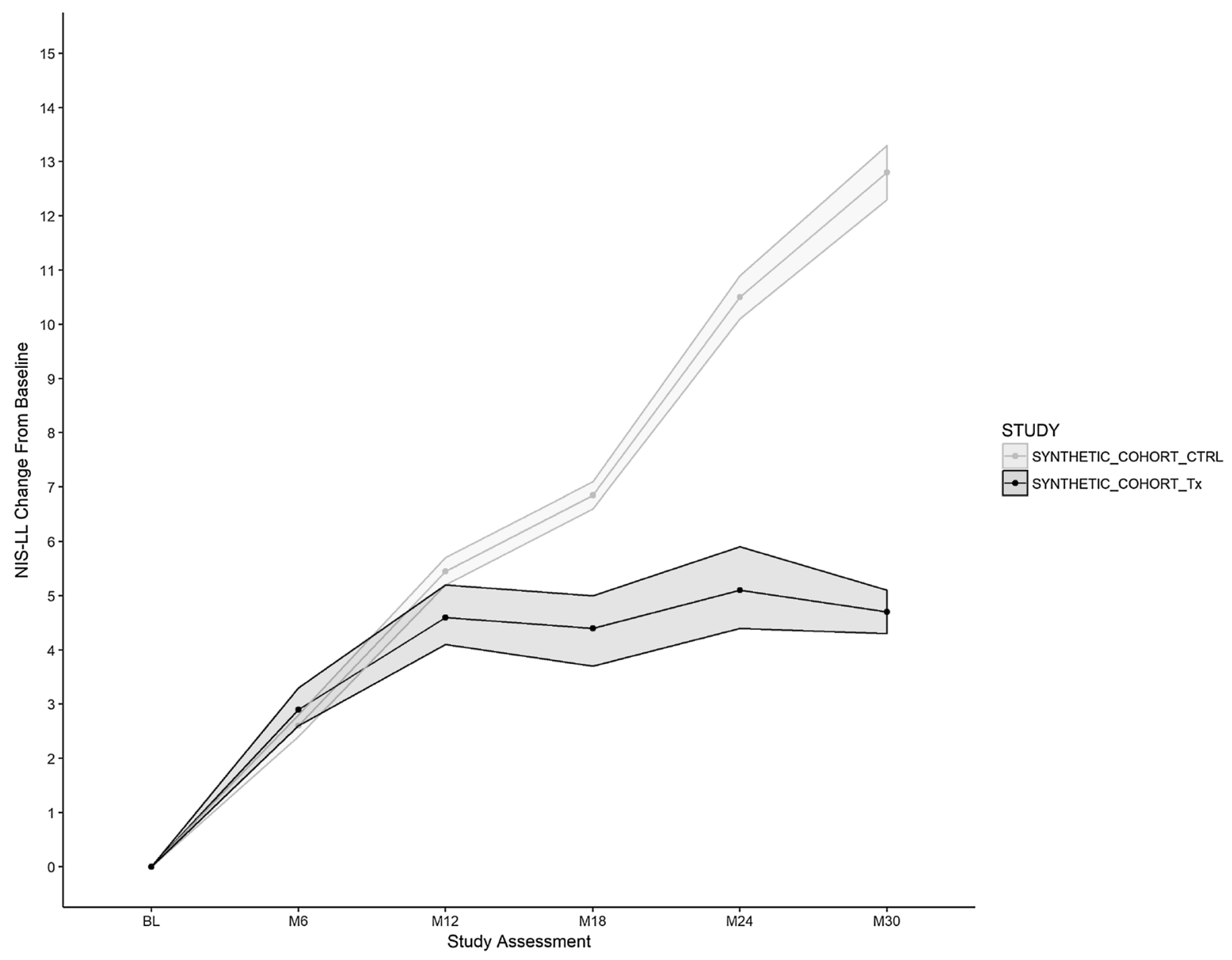

Fig. 5 Synthetic cohort-stratified mean (95\% confidence limits) NIS-LL change from baseline trend. $B L$ baseline, $C T R L$ control, $M$ month, NIS-LL Neuropathy Impairment Score-Lower Limb, Tx treatment

not limited to, incorporation of fixed and random study effects, propensity-matching procedures, and the preferable hybrid of these approaches (i.e., doubly robust propensity weighting). With access to individual patient characteristics and outcomes, more sophisticated statistical techniques and models can also be applied to achieve a greater understanding of clinical outcomes by using a unified process that adjusts for baseline and changes over time. By better characterizing the natural history of untreated hATTR-PN cases and the relative benefit of tafamidis treatment, IDA would significantly facilitate clinician-patient communication regarding available treatment regimens and their respective risks and benefits.

\section{CONCLUSION}

Beyond the registration trial, evidence published to date on the natural history, disease progression, and tafamidis treatment outcomes associated with hATTR-PN has demonstrated some heterogeneity and has been derived from studies with modest sample sizes (due to the low prevalence of this disease). IDA and synthetic cohorts are a technique that can be used to analyze myriad studies with shared features to increase the precision of the characterization of hATTR-PN treatment outcomes. In so doing, modest samples can be aggregated to form large cohorts from which increased precision of inference may be obtained. As this is a proof of 


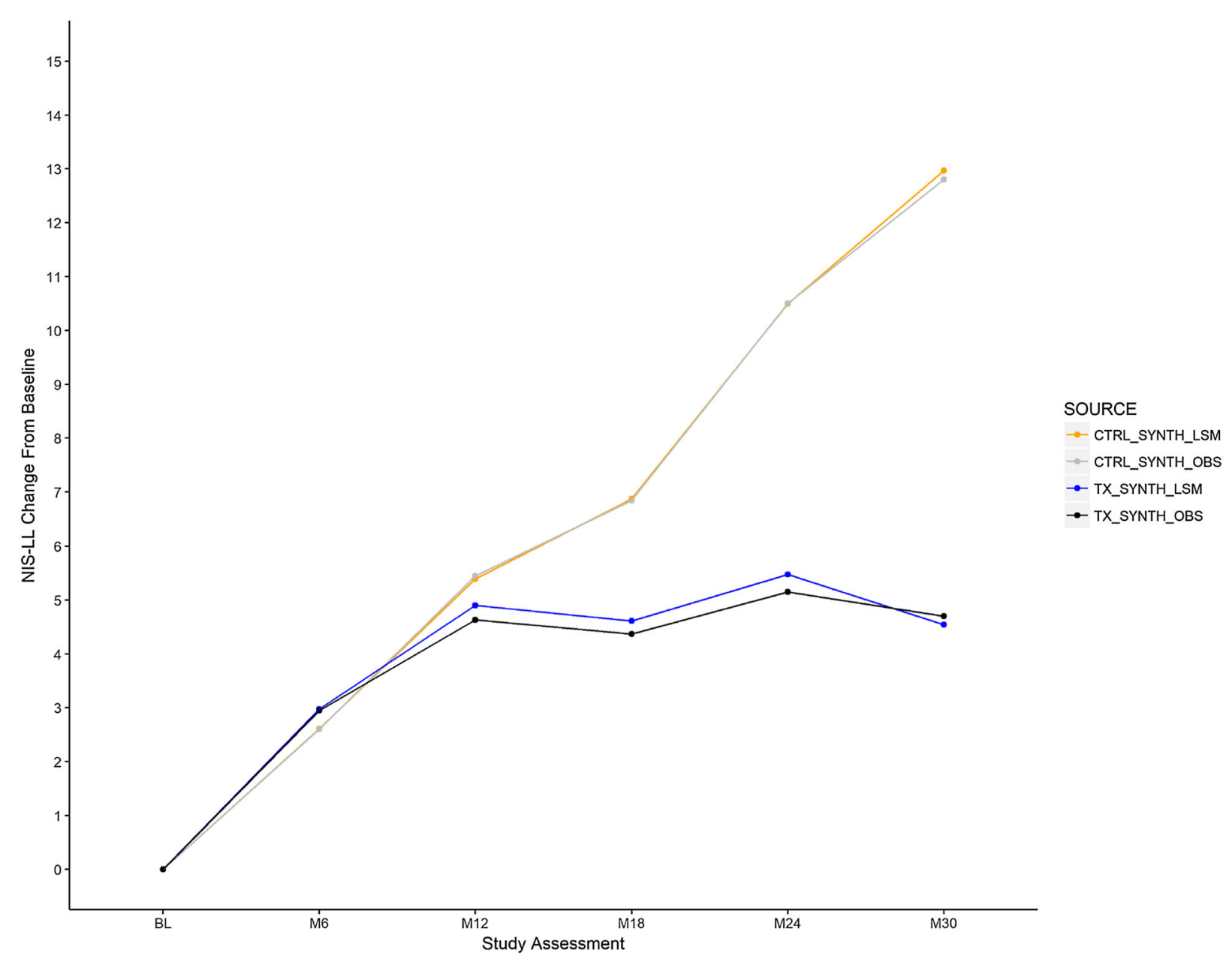

Fig. 6 Treatment arm-stratified observed synthetic cohort means versus MMRM estimated LSMs. $B L$ baseline, CTRL control, LSM least squares mean, $M$ month,

concept for the application of IDA to patientlevel progression data in hATTR-PN, no definite conclusions about the effectiveness of tafamidis can be made from these results. Rather, one can only conclude from the evidence presented herein whether IDA is a method that may be useful in the future for characterizing disease progression and drug effectiveness in a larger cohort using patient-level data.

\section{ACKNOWLEDGEMENTS}

Funding. Sponsorship for this research and article processing fees were funded by Pfizer,
MMRM mixed model for repeated measures, NIS-LL Neuropathy Impairment Score-Lower Limb, OBS observed synthetic cohort, $T X$ treatment

Inc. (New York, USA). All authors had full access to all of the data in this study and take complete responsibility for the integrity of the data and accuracy of the data analysis.

Medical Writing and/or Editorial Assistance. Editorial assistance in the preparation of this article was provided by Catherine A. O'Connor and Caroline Seo of Pharmerit International. Support for this assistance was funded by Pfizer, Inc.

Authorship. All named authors meet the International Committee of Medical Journal Editors (ICMJE) criteria for authorship for this article, take responsibility for the integrity of 
the work as a whole, and have given their approval for this version to be published.

Disclosures. This research was supported by Pfizer, Inc. Marc F. Botteman is a shareholder of Pharmerit International. Daniel Serrano is an employee of Pharmerit International. Christopher B. Atzinger is an employee of Pharmerit International. Marc F. Botteman is an employee of Pharmerit International. Pharmerit received funding from Pfizer, Inc., for study design, execution, analysis, and manuscript development.

Compliance with Ethics Guidelines. This research is based on previously conducted studies and does not contain any studies with human participants or animals performed by any of the authors.

Data availability. All the data used in the analyses are presented in Tables 2 and 3.

Open Access. This article is distributed under the terms of the Creative Commons Attribution-NonCommercial 4.0 International License (http://creativecommons.org/licenses/ by-nc/4.0/), which permits any noncommercial use, distribution, and reproduction in any medium, provided you give appropriate credit to the original author(s) and the source, provide a link to the Creative Commons license, and indicate if changes were made.

\section{REFERENCES}

1. Benson MD. The hereditary amyloidoses. Best Pract Res Clin Rheumatol. 2003;17(6):909-27. https:// doi.org/10.1016/j.berh.2003.09.001.

2. Hund E, Linke RP, Willig F, Grau A. Transthyretinassociated neuropathic amyloidosis.Pathogenesis and treatment. Neurology. 2001;56(4):431-5.

3. Schmidt $\mathrm{HH}$, Waddington-Cruz M, Botteman MF, et al. Estimating the global prevalence of transthyretin familial amyloid polyneuropathy. Muscle Nerve. 2017. https://doi.org/10.1002/mus.26034.

4. Connors LH, Richardson AM, Theberge R, Costello CE. Tabulation of transthyretin (TTR) variants as of 1/1/2000. Amyloid. 2000;7(1):54-69.
5. Coelho T, Merlini G, Bulawa CE, et al. Mechanism of action and clinical application of Tafamidis in hereditary transthyretin amyloidosis. Neurol Ther. 2016;5(1):1-25. https://doi.org/10.1007/s40120016-0040-X.

6. Bekircan-Kurt CE, Gunes N, Yilmaz A, Erdem-Ozdamar S, Tan E. Three Turkish families with different transthyretin mutations. Neuromuscular Disord. 2015;25(9):686-92. https://doi.org/10. 1016/j.nmd.2015.05.010.

7. Bulawa CE, Connelly S, Devit M, et al. Tafamidis, a potent and selective transthyretin kinetic stabilizer that inhibits the amyloid cascade. Proc Natl Acad Sci USA. 2012;109(24):9629-34. https://doi.org/10. 1073/pnas. 1121005109 .

8. Reines JB, Vera TR, Martin MU, et al. Epidemiology of transthyretin-associated familial amyloid polyneuropathy in the Majorcan area: Son Llatzer Hospital descriptive study. Orphanet J Rare Dis. 2014;9:29. https://doi.org/10.1186/1750-1172-929.

9. Benson MD, Kincaid JC. The molecular biology and clinical features of amyloid neuropathy. Muscle Nerve. 2007;36(4):411-23. https://doi.org/10.1002/ mus. 20821.

10. Coelho T, Maia LF, da Silva AM, et al. Long-term effects of tafamidis for the treatment of transthyretin familial amyloid polyneuropathy. J Neurol. 2013;260(11):2802-14. https://doi.org/10.1007/ s00415-013-7051-7.

11. Keohane D, Schwartz J, Gundapaneni B, Stewart M, Amass L. Tafamidis reduces disease progression in patients with transthyretin familial amyloid polyneuropathy: supportive post hoc analyses of a pivotal trial. Orphanet J Rare Dis. 2015;10(Suppl 1):P11. https://doi.org/10.1186/1750-1172-10-s1p11.

12. Barroso FA, Judge DP, Ebede B, et al. Long-term safety and efficacy of tafamidis for the treatment of hereditary transthyretin amyloid polyneuropathy: results up to 6 years. Amyloid. 2017;24(3):194-204. https://doi.org/10.1080/13506129.2017.1357545.

13. Keohane D, Schwartz J, Gundapaneni B, Stewart M, Amass L. Tafamidis delays disease progression in patients with early stage transthyretin familial amyloid polyneuropathy: additional supportive analyses from the pivotal trial. Amyloid. 2017;24(1):30-6. https://doi.org/10.1080/ 13506129.2017.1301419.

14. Merlini G, Plante-Bordeneuve V, Judge DP, et al. Effects of tafamidis on transthyretin stabilization and clinical outcomes in patients with non-Val30Met transthyretin amyloidosis. J Cardiovasc Transl 
Res. 2013;6(6):1011-20. https://doi.org/10.1007/ s12265-013-9512-x.

15. Waddington Cruz M, Amass L, Keohane D, et al. Early intervention with tafamidis provides longterm (5.5-year) delay of neurologic progression in transthyretin hereditary amyloid polyneuropathy. Amyloid. 2016;23(3):178-83. https://doi.org/10. 1080/13506129.2016.1207163.

16. Gundapaneni BK, Sultan MB, Keohane DJ, Schwartz JH. Tafamidis delays neurological progression comparably across Val30Met and non-Val30Met genotypes in transthyretin familial amyloid polyneuropathy. Eur J Neurol. 2017. https://doi. org/10.1111/ene.13510.

17. Ando Y, Coelho T, Berk JL, et al. Guideline of transthyretin-related hereditary amyloidosis for clinicians. Orphanet J Rare Dis. 2013;8:31. https:// doi.org/10.1186/1750-1172-8-31.

18. VYNDAQEL [summary of opinion-initial authorisation] [online]. London: European Medicines Agency 2011-21-July Contract No.: EMA/CHMP/ $546201 / 2011$.

19. Cortese A, Vita G, Luigetti M, et al. Monitoring effectiveness and safety of Tafamidis in transthyretin amyloidosis in Italy: a longitudinal multicenter study in a non-endemic area. J Neurol. 2016;263(5):916-24. https://doi.org/10.1007/ s00415-016-8064-9.

20. Lozeron $\mathrm{P}$, Theaudin M, Mincheva Z, et al. Effect on disability and safety of Tafamidis in late onset of Met30 transthyretin familial amyloid polyneuropathy. Eur J Neurol. 2013;20(12):1539-45. https://doi.org/10.1111/ene.12225.

21. Plante-Bordeneuve V, Gorram F, Salhi H, et al. Long-term treatment of transthyretin familial amyloid polyneuropathy with tafamidis: a clinical and neurophysiological study. J Neurol. 2017;264(2):268-76. https://doi.org/10.1007/ s00415-016-8337-3.
22. Curran PJ, Hussong AM. Integrative data analysis: the simultaneous analysis of multiple data sets. Psychol Methods. 2009;14(2):81.

23. Coelho T, Maia LF, Martins da Silva A, et al. Tafamidis for transthyretin familial amyloid polyneuropathy: a randomized, controlled trial. Neurology. 2012;79(8):785-92. https://doi.org/10.1212/WNL. 0b013e3182661eb1.

24. Wickham H. ggplot2: Elegant graphics for data analysis. New York: Springer; 2009.

25. SAS version 9.4. SAS Institute: Cary, NC, USA; 2013.

26. Adams D, Coelho T, Obici L, et al. Rapid progression of familial amyloidotic polyneuropathy: a multinational natural history study. Neurology. 2015;85(8):675-82. https://doi.org/10.1212/WNL. 0000000000001870 .

27. Ericzon BG, Wilczek HE, Larsson M, et al. Liver transplantation for hereditary transthyretin amyloidosis: after 20 years still the best therapeutic alternative? Transplantation. 2015;99(9):1847-54. https://doi.org/10.1097/TP.0000000000000574.

28. Kerschen P, Plante-Bordeneuve V. Current and future treatment approaches in transthyretin familial amyloid polyneuropathy. Curr Treat Options Neurol. 2016;18(12):53. https://doi.org/10. 1007/s11940-016-0436-z.

29. Suhr OB, Larsson M, Ericzon BG, Wilczek HE, FAPWTR's investigators. Survival after transplantation in patients with mutations other than Val30Met: extracts from the FAP world transplant registry. Transplantation. 2016;100(2):373-81. https://doi.org/10.1097/TP.0000000000001021.

30. Hofer SM, Piccinin AM. Integrative data analysis through coordination of measurement and analysis protocol across independent longitudinal studies. Psychol Methods. 2009;14(2):150. 